\title{
Magnetic properties of iron cluster/chromium matrix nanocomposites
}

\begin{tabular}{|c|c|c|}
\hline Letter & & Open Access \\
\hline Address: & Beilstein J. Nanotechnol. 2015, 6, 1158-1163. & \\
\hline $\begin{array}{l}{ }^{1} \text { Institute of Nanotechnology, Karlsruhe Institute of Technology (KIT), } \\
76344 \text { Eggenstein-Leopoldshafen, Germany and }{ }^{2} \text { KIT-TUD Joint }\end{array}$ & doi:10.3762/bjnano.6.117 & \\
\hline Research Laboratory Nanomaterials, Technische Universität & Received: 27 February 2015 & \\
\hline \multirow[t]{2}{*}{ Darmstadt (TUD), 64287 Darmstadt, Germany } & Accepted: 15 April 2015 & \\
\hline & Published: 13 May 2015 & \\
\hline \multicolumn{3}{|l|}{ Email: } \\
\hline Arne Fischer ${ }^{*}$ - arne.fischer@kit.edu & Associate Editor: P. Ziemann & \\
\hline${ }^{*}$ Corresponding author & $\begin{array}{l}\text { (C) } 2015 \text { Fischer et al; licensee Beilstein-Institut. } \\
\text { License and terms: see end of document. }\end{array}$ & \\
\hline Keywords: & & \\
\hline
\end{tabular}

\begin{abstract}
A custom-designed apparatus was used for the fine-tuned co-deposition of preformed Fe clusters into antiferromagnetic Cr matrices. Three series of samples with precisely defined cluster sizes, with accuracy to a few atoms, and controlled concentrations were fabricated, followed by a complete characterization of structure and magnetic performance. Relevant magnetic characteristics, reflecting the ferromagnetic/antiferromagnetic coupling between Fe clusters and the $\mathrm{Cr}$ matrix, i.e., blocking temperature, coercivity field, and exchange bias were measured and their dependence on cluster size and cluster concentration in the matrix was analyzed. It is evident that the blocking temperatures are clearly affected by both the cluster size and their concentration in the Cr matrix. In contrast the coercivity shows hardly any dependence on size or inter-cluster distance. The exchange bias was found to be strongly sensitive to the cluster size but not to the inter-cluster distances. Therefore, it was concluded to be an effect that is purely localized at the interfaces.
\end{abstract}

\section{Introduction}

Today's metallic alloys are prepared by using complex thermomechanical treatment steps, i.e., quenching, annealing combined with plastic deformation, in order to obtain the multicomponent multiphase structures optimized for advanced structural and functional applications [1]. Besides the pathways used during the preparation of the alloys, their final nano- and microstructure is determined strongly by the phase diagram limiting the extent of deviation from the well-defined thermodynamic equilibrium, which, for example, determines the volume

fraction of precipitates or second phase particles and the composition of the matrix phase. Oxide dispersion strengthened alloys (ODS) are exceptions, as the distribution of oxide particles in the metallic matrix can be modified without the above mentioned constraints as the processing is done by mechanical alloying, not via the melt route followed by thermo-mechanical treatments. In metallic multiphase alloys, however, the ranges of precipitate sizes and the width of their distributions, as well as the chemical compositions of the precipitates and the matrix 
are severely limited by the thermodynamics of the alloy systems. Therefore, in any case the potential of alloy design will remain limited as long as thermo-mechanical treatment is employed for processing of alloys.

Simultaneous deposition of preformed clusters with pre-selected sizes ranging from a few atoms to thousands of atoms and of an atomic beam of another element onto a substrate opens a way to overcome this dilemma. There is a rich literature on the synthesis of charged clusters of basically any element and many alloy systems and their transfer into an ultra-high vacuum system (UHV). The deposition of the charged clusters onto substrates can be performed with variable impact energies. Such a process opens a new way for the synthesis of cluster-based alloys, i.e., multiphase alloys with extreme control of the fraction of clusters inside a matrix consisting of another element or alloy system. For the alloy system $\mathrm{Fe} / \mathrm{Ag}$ it has been shown that full control over the overall composition of the two immiscible elements can be achieved [2]. One of the scopes of the experiments with $\mathrm{Fe} / \mathrm{Ag}$ was to study the characteristics of the embedded Fe clusters. Since Ag is diamagnetic no noteworthy magnetic interaction takes place between matrix and the ferromagnetic clusters and it was possible to gain information about, e.g., the size of the embedded clusters via magnetic measurements. The intention of the present work is to go one step further to a more complex cluster/matrix system and to substitute the passive Ag matrix with a functional one, e.g., antiferromagnetic (AFM) Cr, leading to additional effects: At the interface between the ferromagnetic (FM) and the antiferromagnetic (AFM) phases a spin exchange coupling occurs and a part of the magnetic moments of the FM phase become pinned. This results in an increased magnetic anisotropy manifesting itself as an exchange bias effect (EB) [3]. The EB appears as a horizontal shift of the magnetization loops, the EB field $H_{\mathrm{eb}}$, and is usually accompanied by an increase of coercivity $\left(H_{\mathrm{c}}\right)$ and of the blocking temperature $\left(T_{\mathrm{B}}\right)$. The EB was first described by Meiklejohn and Bean in 1956 [4]. They investigated clusters with a FM cobalt core and an AFM cobalt oxide ( $\mathrm{CoO})$ shell and consequently observed the characteristic horizontal shift of the hysteresis loops recorded after field cooling the samples from temperatures above the Néel temperature $\left(T_{\mathrm{N}}\right)$ of $\mathrm{CoO}$.

Since its discovery the EB has been observed in numerous FM/AFM combinations such as core/shell clusters [5,6], thin film systems [7,8] and also cluster/matrix combinations [9-11]. So far, most of the research has been focused on thin film systems due to their commercial importance for reading heads in magnetic data storage [12]. Since many difficulties arise in fabricating FM cluster/AFM matrix systems in a strictly controlled way there are fewer studies compared to thin films.
In principle there are two main approaches to the fabrication of FM cluster/AFM matrix systems. The first is to co-evaporate several materials or to chemically produce a compound in a first step and to induce the formation of FM precipitates in a leftover AFM matrix in a second step (e.g., by heating) $[13,14]$. The drawback of this approach is the lack of serious control over the size and density of the precipitates in the matrix. The alternative is to co-deposit preformed FM clusters (e.g., by inert gas-condensation) and AFM matrices [9-11]. In that case the cluster size can be well-defined and, having control over the exact deposition rates of the clusters and the matrix, the amount of clusters can also be exactly adjusted. However, to date, only a few studies on the EB in cluster/matrix systems have been published, most of them being based on a very limited number of samples.

In this paper, a rather comprehensive study of the magnetic characteristics in the system of preformed Fe clusters embedded in $\mathrm{Cr}$ matrices is presented. It is based on the largest series of samples (20) for any FM/AFM cluster/matrix combination reported in literature. Due to the large amount of samples representing three different cluster sizes and a broad range of cluster concentrations in the matrix, combined with a high degree of control over the experimental conditions, the effects of the two critical parameters, cluster size and density in the matrix on $T_{\mathrm{B}}$, $H_{\mathrm{c}}$ and $H_{\mathrm{eb}}$ could be clearly shown. The system $\mathrm{Fe}_{x} / \mathrm{Cr}$ is a perfect model system: being just based on two components $(\mathrm{Cr}$ is an AFM element), it avoids the pitfall of compositional variations in the AFM (e.g., only partially oxidized $\mathrm{CoO}$ ) which may lead to additional, unwanted effects.

\section{Results and Discussion}

The samples were prepared in a newly developed UHV cluster ion beam deposition apparatus, which is described elsewhere [2]. Fe clusters are produced in a Haberland-type magnetron sputtering/gas aggregation cluster source. Extracted anions are accelerated by electrostatic lenses and mass-separated in a $90^{\circ}$ sector magnet. The mass resolution depends on the cluster size and can be estimated to be better than $1 / 10$ for the utilized clusters. Prior to deposition the clusters are decelerated to $50 \mathrm{eV}$ and then soft-landed on a silicon substrate with a native oxide layer (still conducting). To avoid migration and agglomeration of the clusters the substrate is cooled with liquid nitrogen during deposition. The $\mathrm{Cr}$ matrix is co-deposited by using an effusion cell. The flux of matrix material is monitored by a quartz crystal thickness monitor and the cluster flux by counting charges impinging on the sample area with a picoamperemeter (the clusters are singly charged). Counting the charges in combination with the known cluster mass from the mass separation the amount of deposited cluster material can be precisely derived. In order to minimize contamination with, e.g., oxygen the pres- 
sure in the deposition chamber is maintained in the $10^{-9}$ mbar range during the deposition.

$\mathrm{Fe}_{x} / \mathrm{Cr}$ samples consist of the already mentioned Si substrate with a native oxide layer, a $10 \mathrm{~nm} \mathrm{Cr}$ base layer, the Fe cluster/ Cr matrix layer, a $10 \mathrm{~nm} \mathrm{Cr}$ top layer and a $10 \mathrm{~nm} \mathrm{Au} \mathrm{film} \mathrm{as}$ oxidation protection. This geometry makes sure that the Fe clusters are in contact with $\mathrm{Cr}$ only and no oxidation takes place after deposition. To allow for a detailed comparative study of the magnetic characteristics of the samples the absolute amount of deposited Fe is the same for all samples, namely a $6 \mathrm{~nm}$ equivalent film thickness of clusters, and the Fe cluster concentration was adjusted by the amount of deposited $\mathrm{Cr} . \mathrm{Fe}_{x} / \mathrm{Cr}$ samples were produced with Fe cluster sizes of 500, 1000 and 2000 atoms per cluster, corresponding to cluster diameters of 2.3, 2.8 and $3.6 \mathrm{~nm}$, respectively and cluster volume fractions ranging from 2 to $50 \mathrm{vol} \%$. For the three cluster sizes $(500$, 1000 and 2000 atoms/cluster) the aforementioned deposition energy of $50 \mathrm{eV}$ results in $0.1,0.05$ and $0.025 \mathrm{eV} /$ atom, respectively, which is clearly below the binding energy per atom. Therefore, fragmentation as well as pronounced deformation of the clusters during landing can be excluded [15].

Figure 1 shows energy-filtered transmission electron microscopy (EFTEM) and scanning transmission electron microscopy (STEM) micrographs of the Fe distribution for a 10 vol \% $\mathrm{Fe}_{1000} / \mathrm{Cr}$ sample, specifically prepared for TEM. To avoid subsequent focused ion beam cutting and possible oxidation, the sample was deposited on a TEM grid covered with a thin amorphous carbon film while the whole sample thickness including top and bottom $\mathrm{Cr}$ layers was just $5 \mathrm{~nm}$. Deposition parameters such as the cluster deposition rate and the sample temperature during deposition were identical with the ones used for the other samples. The EFTEM micrograph clearly shows that the Fe clusters are evenly distributed in the matrix and no significant agglomeration occurs. In the STEM image individual Fe clus-

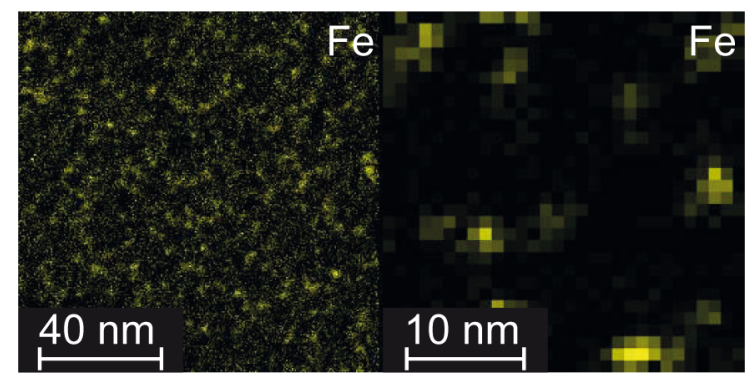

Figure 1: EFTEM (left) and STEM (right) micrographs of a $10 \mathrm{vol} \%$ $\mathrm{Fe}_{1000} / \mathrm{Cr}$ sample prepared on a TEM grid + amorphous carbon film with an Fe cluster equivalent thickness of $0.2 \mathrm{~nm}$. The EFTEM image shows the Fe cluster distribution in the sample and the STEM image individual Fe clusters, it was recorded using EDX and the Fe K signal. ters are clearly visible. Their size can be estimated to be roughly $3 \mathrm{~nm}$ which matches the expected $2.8 \mathrm{~nm}$. Additional diffraction data from TEM (not shown here) revealed that the Fe clusters as well as the $\mathrm{Cr}$ matrix both retain the bec structure as expected.

In the following paragraphs the magnetic properties of the $\mathrm{Fe}$ cluster assemblies in the $\mathrm{Cr}$ matrix are discussed. The magnetic characteristics are extracted from standard zero-field cooled/ field cooled (ZFC/FC) magnetization measurements and magnetic hysteresis loops recorded in a commercial superconducting quantum interference device (SQUID, Quantum Design) magnetometer.

The $\mathrm{ZFC} / \mathrm{FC}$ curves were collected with an applied external magnetic field of $\mu_{0} H=20 \mathrm{mT}$ in a temperature range between 10 and $350 \mathrm{~K}$. The measurement geometry was in-plane, i.e., the external magnetic field was applied parallel to the sample surface (as for all magnetic data presented in this paper).

Figure 2 shows the $T_{\mathrm{B}}$ of the $\mathrm{Fe}_{x} / \mathrm{Cr}$ samples extracted from the $\mathrm{ZFC} / \mathrm{FC}$ curves. At this point it is reasonable to assume that possible interactions between the clusters would not directly depend on the volume fraction of the clusters in the matrix, but on the average distances of neighboring clusters. To approximate this distance for the actually randomly distributed clusters, a body centered cubic (bcc) arrangement of clusters was assumed and the $T_{\mathrm{B}}$ (and subsequent magnetic data) were plotted versus the obtained nearest neighbor distances $D_{\mathrm{NN}}$. The data presented in this way reveal, that $T_{\mathrm{B}}$ is indeed affected both by the size of the embedded Fe clusters as well as $D_{\mathrm{NN}}$. The values of $T_{\mathrm{B}}$ are higher for larger clusters and rise nearly linear (in the investigated region) with decreasing values of $D_{\mathrm{NN}}$ with the linear slope being smaller for larger clusters. Thus, the differences in $T_{\mathrm{B}}$ between the three cluster sizes become distinctly smaller at smaller $D_{\mathrm{NN}}$ (higher volume fraction of the clusters). To minimize the influence of inter-cluster interactions the dependency on the cluster size should be first considered for the larger cluster distances. As a starting point for the analysis one could refer to the simplest model of non-interacting particles with an uniaxial anisotropy in a non-magnetic matrix. Here one would expect a simple proportionality $T_{\mathrm{B}} \propto K_{\text {eff }} V$, where $K_{\text {eff }}$ is an effective anisotropy constant and $V$ the particle volume. Indeed, the measured $T_{\mathrm{B}}$ show some rudimentary size dependence, especially at large $D_{\mathrm{NN}}$, but they do not scale linearly with the cluster size. Also the estimated $K_{\text {eff }}$ of $(0.8-1.3) \times 10^{6} \mathrm{~J} / \mathrm{m}^{3}$ is almost two orders of magnitude bigger than one would expect for clusters with the magnetocrystalline anisotropy of bulk $\alpha$-iron. Both results lead to the conclusion that for the lowest concentration of clusters the effective anisotropy constant is determined by magnetic exchange inter- 
actions with the Cr matrix, which is substantiated by a direct comparison with the Fe clusters embedded in a nonmagnetic $\mathrm{Ag}$ matrix. The $\mathrm{Fe}_{1000}$ clusters with $D_{\mathrm{NN}} \approx 9 \mathrm{~nm}(2 \mathrm{vol} \% \mathrm{Fe})$ were studied earlier and their $T_{\mathrm{B}}$ was about $6 \mathrm{~K}$ [2]. An increase of the $T_{\mathrm{B}}$ by almost one order of magnitude to $53 \mathrm{~K}$, for the $\mathrm{Fe}_{1000}$ clusters deposited in the AFM Cr matrix unambiguously points out the decisive role of FM/AFM exchange coupling in the enhancement of the magnetic anisotropy. For lower $D_{\mathrm{NN}}$ the effect of the particle size on $T_{\mathrm{B}}$ gets even less pronounced, since magnetic inter-particle interactions (e.g., strong dipole-dipole interactions) become dominant.

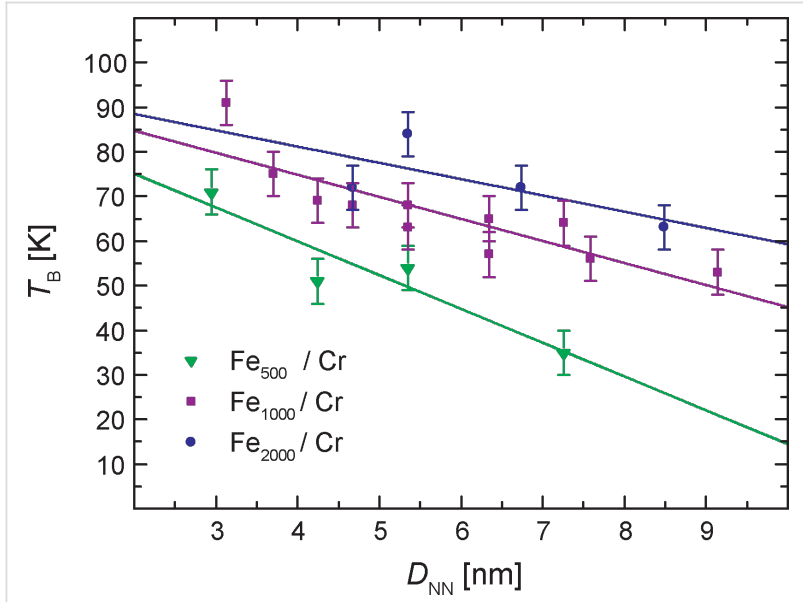

Figure 2: Blocking temperature $T_{\mathrm{B}}$ versus approximated nearest neighbor distances $D_{\mathrm{NN}}$ for $\mathrm{Fe}_{\mathrm{X}} / \mathrm{Cr}$ samples. The solid lines on three series of samples with 500,1000 and 2000 atoms are just guides to the eye. A clear dependence of $T_{\mathrm{B}}$ on the cluster size as well as $D_{\mathrm{NN}}$ is visible.

Hysteresis loops were recorded at $5 \mathrm{~K}$ after field cooling from $350 \mathrm{~K}$, which is above the $T_{\mathrm{N}}$ of $\mathrm{Cr}$ (311 K [16]), in an external magnetic field of $\mu_{0} H=4.5 \mathrm{~T}$. A linear diamagnetic background originating from the Si substrate as well as the Au layers was subtracted. The coercivity $H_{\mathrm{c}}$ of the samples can be derived from the recorded hysteresis loops as $H_{\mathrm{c}}=\left(H_{\mathrm{c}}^{+}-H_{\mathrm{c}}^{-}\right) / 2$, with $H_{\mathrm{c}}^{+}$and $H_{\mathrm{c}}^{-}$being the external field values $\mu_{0} H$ for which the magnetization $M=0$ at the positive and negative branches of the magnetic hysteresis loops respectively. The obtained values of $H_{\mathrm{c}}$ for the three sample series are shown in Figure 3.

In the investigated region $H_{\mathrm{c}}$ shows no clear dependence on the size of the embedded Fe clusters, but rises slightly with decreasing $D_{\mathrm{NN}}$ from roughly 1550 Oe $\left(D_{\mathrm{NN}} \approx 9 \mathrm{~nm}\right)$ to around 2000 Oe $\left(D_{\mathrm{NN}} \approx 3 \mathrm{~nm}\right)$. This behavior shows that $H_{\mathrm{c}}$ mainly depends on the local anisotropy of the Fe clusters and rather weakly rises for smaller $D_{\mathrm{NN}}$ due to extra anisotropy from interactions between the individual Fe clusters. Comparing again the $\mathrm{Fe}_{1000} / \mathrm{Cr}$ sample with $D_{\mathrm{NN}} \approx 9 \mathrm{~nm}(2 \mathrm{vol} \% \mathrm{Fe})$ with the above mentioned $\mathrm{Fe}_{1000} / \mathrm{Ag}$ sample with the same cluster volume frac-

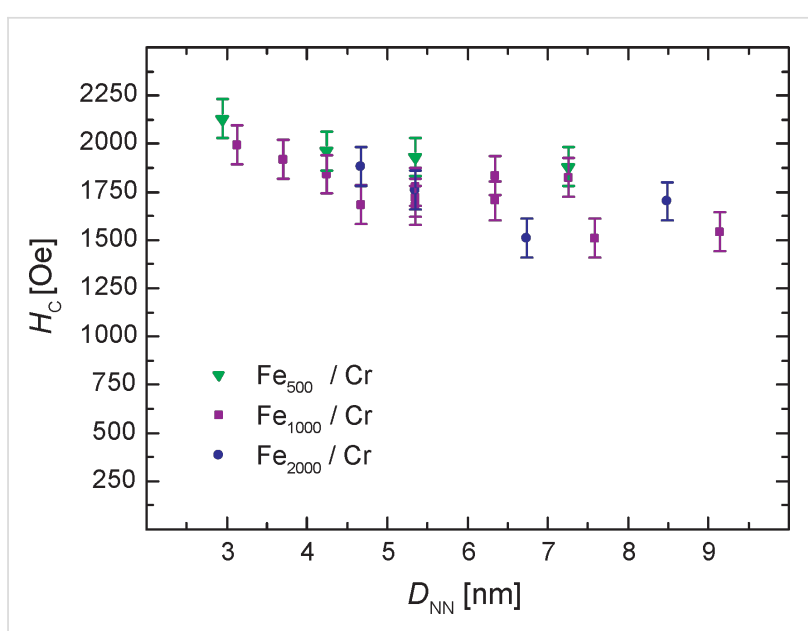

Figure 3: $H_{\mathrm{C}}$ versus $D_{\mathrm{NN}}$ for $\mathrm{Fe}_{\mathrm{x}} / \mathrm{Cr}$ samples. $H_{\mathrm{C}}$ mainly depends on $D_{\mathrm{NN}}$, no clear effect of the cluster size is visible in the investigated region.

tion a distinct rise in $H_{\mathrm{c}}$ from 56 Oe for $\mathrm{Fe}_{1000} / \mathrm{Ag}$ to $1543 \mathrm{Oe}$ for $\mathrm{Fe}_{1000} / \mathrm{Cr}$ is found, underlining again the distinct change of the anisotropy constant $K_{\text {eff }}$ due to the FM/AFM interactions with the Cr matrix.

The horizontal shift of the magnetic hysteresis loops is described by $H_{\mathrm{eb}}=\left(H_{\mathrm{c}}^{+}-H_{\mathrm{c}}^{-}\right) / 2$. Figure 4 shows the values of $H_{\mathrm{eb}}$ extracted from the magnetic hysteresis loops of the different samples. The EB values are basically independent of $D_{\mathrm{NN}}$. The largest series of $\mathrm{Fe}_{1000} / \mathrm{Cr}$ samples exhibits almost linear behavior with a negligible slope of 2.4(4.5) $\mathrm{nm} \cdot \mathrm{Oe}^{-1}$. Therefore, the data can be described within the error with a horizontal line, implying that the volume fraction of the Fe clusters has either no or only little influence on $H_{\mathrm{eb}}$. On the other

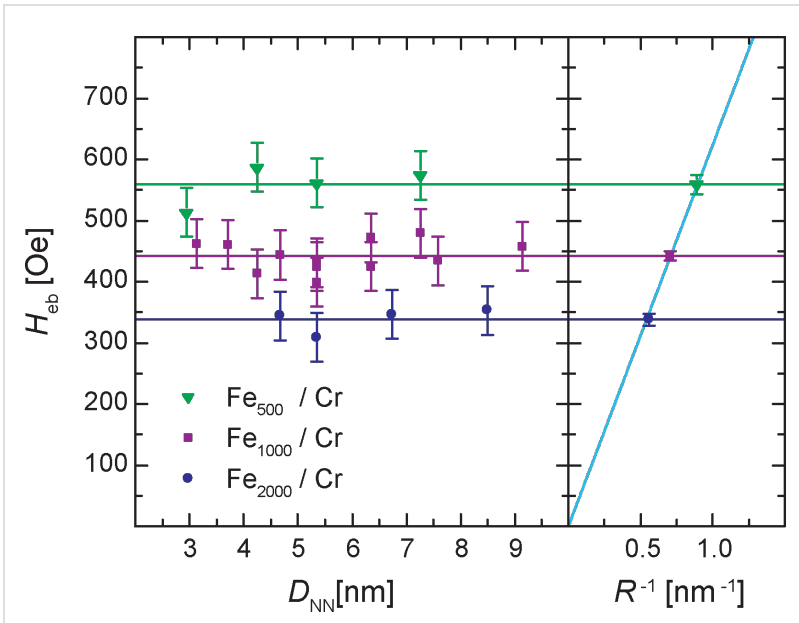

Figure 4: Left: $H_{\mathrm{eb}}$ versus $D_{\mathrm{NN}}$ for the three series of samples with different cluster sizes. $D_{\mathrm{NN}}$ has no effect on $H_{\mathrm{eb}}$, while a pronounced effect is found for the size of the embedded clusters. Right: Average $H_{\text {eb }}$ versus $R^{-1}$ for the three cluster sizes showing a linear relation. 
hand comparing the average $H_{\mathrm{eb}}$ values of the three series with different cluster sizes a pronounced effect of the cluster size on $H_{\mathrm{eb}}$ is clearly visible. Fitting horizontal lines to each series one obtains average values of $H_{\mathrm{eb}}$ of 559(16) Oe, 442(7) Oe and 338(10) Oe for $\mathrm{Fe}_{500} / \mathrm{Cr}, \mathrm{Fe}_{1000} / \mathrm{Cr}$ and $\mathrm{Fe}_{2000} / \mathrm{Cr}$, respectively.

To model the dependence of $H_{\mathrm{eb}}$ on the cluster size one should keep in mind that in first approximation the EB is an interface phenomenon. When the FM Fe clusters are cooled in the external magnetic field down below $T_{\mathrm{N}}$ of the $\mathrm{Cr}$ matrix the clusters lock AFM Cr domains in a certain direction via exchange FM/AFM interactions. The initial orientation of the AFM domains determines the unidirectional anisotropy axis resulting in the shift of the magnetization hysteresis loops. For spherical FM clusters one can assume that FM spins residing on the surface are exchange-coupled to the AFM neighbors with a strength determined by the exchange integral $J$. The FM/AFM interaction keeps all the spins of the cluster along the unidirectional anisotropy axis. During the magnetization switching process the external magnetic field flips the magnetization in the opposite direction. The switching field must overcome the FM/AFM coupling which is proportional to $J$ multiplied by the cluster surface area $\pi R^{2}$, where $R$ is the cluster radius. On the other hand the bigger the total magnetic moment of the cluster (which is proportional to the number of magnetic moments per cluster and, thus, to the volume, i.e., to $R^{3}$ ) the higher is the torque induced by the external magnetic field and the easier is the rotation away from an easy axis. Thus the switching field is proportional to the ratio $J \cdot R^{2} / R^{3}$, which eventually results in $H_{\mathrm{eb}} \propto 1 / R$. Plotting the obtained $H_{\mathrm{eb}}$ values for the three cluster sizes versus $1 / R$ of the clusters (Figure 4 ) and assuming $H_{\mathrm{eb}}=0$ for an infinitely large particle $(1 / R=0)$ a linear relation is found in the investigated region of cluster radii and a linear fit to the data yields a slope of 624(7) $\mathrm{Oe}^{-1} \cdot \mathrm{nm}^{-1}$. This straightforward relation between $H_{\mathrm{eb}}$ and $R$ of the embedded clusters has never been shown to that degree in any FM/AFM cluster/matrix system.

As a comparison, one can look at the closely related core/shell nanoparticles featuring a FM core and an AFM shell. In that case a theoretical study predicted an oscillatory relation of $H_{\mathrm{eb}}$ and $R$ [17]. On the other hand one can also refer to thin film systems composed of a FM and an AFM layer. It was shown by Restrepo-Parra et. al. [18] that $H_{\mathrm{eb}} \propto 1 / D^{m}$, with $m \approx 1$ and $D$ being the thickness of the FM layer. This result nicely supports our finding, since it became clear in both studies that $H_{\mathrm{eb}}$ is basically proportional to the surface to volume or interface to volume ratio of the FM part of the system.

$\mathrm{Fe}_{x} / \mathrm{Cr}$ was already studied in a previous publication by Qureshi et al. [11]. It is based on three samples with different volume fractions of clusters of a single size $(\approx 340$ atoms/cluster $)$ and amongst others they report on $H_{\mathrm{c}}$ and $H_{\mathrm{eb}}$ that were both found to rise with rising volume fraction of the clusters. Compared to the results shown in the present study the behavior of $H_{\mathrm{c}}$ exhibits a similar trend with the absolute values being three to five times lower. For $H_{\mathrm{eb}}$ the values are between two and five times lower than the lowest $H_{\mathrm{eb}}$ observed here (310 Oe). In addition they show a dependence on the volume fraction of the clusters, which is not validated in the present study. Of course these discrepancies cannot be easily addressed, but it needs to be stated that it was found in first test experiments that a high degree of control over the deposition parameters is of utmost importance for the consistency of the obtained data. For instance, in trial experiments the sample temperature varied during the deposition from sample to sample due to different evaporator temperatures or erratic thermal contact of the sample to the sample holder which resulted in quite different magnetic characteristics. Only after cooling the samples with liquid nitrogen during deposition and gluing the samples to the sample holders with silver glue as well as keeping the deposition times similar for all samples it was possible to get reproducible and consistent results.

In conclusion, by using a dedicated UHV cluster-deposition apparatus we fabricated in a highly controllable way series of samples with Fe clusters embedded in Cr matrices. Subsequently, the magnetic characteristics of 20 samples with three different cluster sizes and varied cluster volume fractions were studied to determine their relevant parameters: $T_{\mathrm{B}}, H_{\mathrm{c}}$ and $H_{\mathrm{eb}}$. While $T_{\mathrm{B}}$ is found to be dependent on the size of the embedded clusters as well as on the average distance between neighboring clusters $D_{\mathrm{NN}}, H_{\mathrm{c}}$ is found to depend rather weakly on $D_{\mathrm{NN}}$. The exchange bias field $H_{\mathrm{eb}}$ responds to the size of the embedded clusters $\left(H_{\mathrm{eb}} \propto 1 / R\right)$ but is actually not depending on the cluster concentration. With this observation one arrives at the conclusion that the exchange bias effect is a rather local effect limited to a few layers of the AFM Cr surrounding the FM Fe cluster.

\section{Acknowledgements}

The authors are grateful for a generous equipment grant for the cluster deposition system from the federal state of Hessen (Germany).

\section{References}

1. Padmanabhan, K. A.; Gleiter, H. Beilstein J. Nanotechnol. 2014, 5 , 1603-1615. doi:10.3762/bjnano.5.172

2. Fischer, A.; Kruk, R.; Hahn, H. Rev. Sci. Instrum. 2015, 86, 023304. doi:10.1063/1.4908166

3. Skumryev, V.; Stoyanov, S.; Zhang, Y.; Hadjipanayis, G.; Givord, D.; Nogués, J. Nature 2003, 423, 850-853. doi:10.1038/nature01687 
4. Meiklejohn, W.; Bean, C. Phys. Rev. 1957, 105, 904-913. doi:10.1103/PhysRev.105.904

5. Peng, D.; Sumiyama, K.; Hihara, T.; Yamamuro, S.; Konno, T. Phys. Rev. B 2000, 61, 3103-3109. doi:10.1103/PhysRevB.61.3103

6. Binns, C.; Qureshi, M. T.; Peddis, D.; Baker, S. H.; Howes, P. B.; Boatwright, A.; Cavill, S. A.; Dhesi, S. S.; Lari, L.; Kröger, R.; Langridge, S. Nano Lett. 2013, 13, 3334-3339. doi:10.1021/nl401587t

7. Escorcia-Aparicio, E.; Choi, H.; Ling, W.; Kawakami, R.; Qiu, Z. Phys. Rev. Lett. 1998, 81, 2144-2147. doi:10.1103/PhysRevLett.81.2144

8. Ciprian, R.; Carbucicchio, M.; Palombarini, G. IEEE Trans. Magn. 2010, 46, 432-435. doi:10.1109/TMAG.2009.2032266

9. Tobia, D.; Winkler, E.; Zysler, R. D.; Granada, M.; Troiani, H. E.; Fiorani, D. J. Appl. Phys. 2009, 106, 103920. doi:10.1063/1.3259425

10. Kuerbanjiang, B.; Wiedwald, U.; Haering, F.; Biskupek, J.; Kaiser, U.; Ziemann, P.; Herr, U. Nanotechnology 2013, 24, 455702. doi:10.1088/0957-4484/24/45/455702

11. Qureshi, M.; Baker, S.; Binns, C.; Roy, M.; Laureti, S.; Fiorani, D.; Peddis, D. J. Magn. Magn. Mater. 2015, 378, 345-352. doi:10.1016/j.jmmm.2014.11.065

12. Dieny, B.; Speriosu, V.; Parkin, S.; Gurney, B.; Wilhoit, D.; Mauri, D. Phys. Rev. B 1991, 43, 1297-1300. doi:10.1103/PhysRevB.43.1297

13. Shoemaker, D. P.; Grossman, M.; Seshadri, R. J. Phys.: Condens. Matter 2008, 20, 195219. doi:10.1088/0953-8984/20/19/195219

14. Tian, Z. M.; Yuan, S. L.; Yin, S. Y.; Liu, L.; He, J. H.; Duan, H. N.; Li, P.; Wang, C. H. Appl. Phys. Lett. 2008, 93, 222505. doi:10.1063/1.3039071

15. Haberland, H.; Insepov, Z.; Moseler, M. Phys. Rev. B 1995, 51, 11061-11067. doi:10.1103/PhysRevB.51.11061

16. Fawcett, E. Rev. Mod. Phys. 1988, 60, 209-283. doi:10.1103/RevModPhys.60.209

17. Iglesias, O.; Batlle, X.; Labarta, A. J. Phys. D: Appl. Phys. 2008, 41, 134010. doi:10.1088/0022-3727/41/13/134010

18. Restrepo-Parra, E.; Restrepo, J.; Jurado, J. F.; Vargas-Hernandez, C.; Riano-Rojas, J. C. IEEE Trans. Magn. 2009, 45, 5180-5183. doi:10.1109/TMAG.2009.2031080

\section{License and Terms}

This is an Open Access article under the terms of the Creative Commons Attribution License (http://creativecommons.org/licenses/by/2.0), which permits unrestricted use, distribution, and reproduction in any medium, provided the original work is properly cited.

The license is subject to the Beilstein Journal of Nanotechnology terms and conditions: (http://www.beilstein-journals.org/bjnano)

The definitive version of this article is the electronic one which can be found at: $\underline{\text { doi:10.3762/bjnano.6.117 }}$ 